$\mathrm{J}$ o u r n a l of

Mathematics

and Applications

JMA No 42, pp 21-33 (2019)

\title{
On Some Fixed Point Theorems for Expansive Mappings in Dislocated Cone Metric Spaces with Banach Algebras
}

\author{
Abba Auwalu, Evren Hinçal and Lakshmi Narayan Mishra*
}

\begin{abstract}
In this paper, we introduced the notion of generalized expansive mappings in dislocated cone metric spaces with Banach algebras. Furthermore, we prove some fixed point theorems for generalized expansive mappings in dislocated cone metric spaces with Banach algebras without the assumption of normality of cones. Moreover, we give an example to elucidate our result. Our results are significant extension and generalizations of many recent results in the literature.
\end{abstract}

AMS Subject Classification: 47H10, 54H25.

Keywords and Phrases: Dislocated cone metric space over Banach algebras; Expansive mapping; Fixed point; $c$-sequence.

\section{Introduction}

The concept of cone metric space was introduced by Huang and Zhang [9]. They supplanted the set of real numbers in metric space by a complete normed space and proved some fixed point results for different contractive conditions in such a space. Later on, Liu and $\mathrm{Xu}$ [13] introduced the notion of cone metric space over Banach algebras by supplanting the complete normed space in cone metric space with Banach algebras and proved that cone metric space over Banach algebras are not equivalent to metric space in terms of existence of the fixed points of mappings. Subsequently, many authors established interesting and significant results in a cone metric space over Banach algebras (see [20], [7], [8]). In 2017, George et al. [6] introduced the notion of dislocated cone metric space over Banach algebras as a generalization of cone metric space over Banach algebras and proved some fixed point results for Banach, Kannan

COPYRIGHT (c) by Publishing House of Rzeszów University of Technology P.O. Box 85, 35-959 Rzeszów, Poland 
and Perov type contractive conditions in such a space. Very recently, Jiang et al. [11] introduced the concept of expansive mapping defined on cone metric space over Banach algebras and proved some fixed point results for such mapping. In this work, we use the concept of expansive mapping defined on dislocated cone metric space over Banach algebras and prove some fixed point theorems. Our results unify, complement and/or generalized the recent results of [11, 2, 10, 1, 3, 19], and many others, that will be useful in dynamic programming and integral equation, (see; [4] - [15] and references therein).

\section{Preliminaries}

In this section, we recall some definitions and results needed in the sequel.

Definition 2.1. ([18]) A Banach algebra $\mathcal{A}$ is a real Banach space in which an operation of multiplication is defined subject to the following properties for all $p, q, r \in \mathcal{A}$, $\lambda \in \mathbb{R}$

1. $(p q) r=p(q r)$,

2. $p(q+r)=p q+p r$ and $(p+q) r=p r+q r$,

3. $\lambda(p q)=(\lambda p) q=p(\lambda q)$,

4. $\|p q\| \leq\|p\|\|q\|$.

A subset $\mathcal{K}$ of a Banach algebra $\mathcal{A}$ is called a cone (see [13]) if

1. $\mathcal{K}$ is nonempty closed and $\{\theta, e\} \subset \mathcal{K}$;

2. $\alpha \mathcal{K}+\beta \mathcal{K} \subset \mathcal{K}$ for all nonnegative real numbers $\alpha, \beta$;

3. $\mathcal{K}^{2}=\mathcal{K} \mathcal{K} \subset \mathcal{K}$

4. $\mathcal{K} \cap(-\mathcal{K})=\{\theta\}$,

where $\theta$ and $e$ denote the zero and unit elements of the Banach algebra $\mathcal{A}$, respectively. For a given cone $\mathcal{K} \subset \mathcal{A}$, we write $z \preccurlyeq y$ if and only if $y-z \in \mathcal{K}$, where $\preccurlyeq$ is a partial order relation defined on $\mathcal{K}$. Also, $x \ll y$ will stand for $y-x \in$ int $\mathcal{K}$, where $i n t \mathcal{K}$ denotes the interior of $\mathcal{K}$. If $i n t \mathcal{K} \neq \varnothing$ then $\mathcal{K}$ is called a solid cone.

Definition 2.2. ([6]) Let $\mathcal{Z}$ be a nonempty set. Suppose that $\rho: \mathcal{Z} \times \mathcal{Z} \rightarrow \mathcal{A}$ be a mapping satisfying the following conditions:

$\left(D_{1}\right) \quad \theta \preccurlyeq \rho(z, y)$ for all $z, y \in \mathcal{Z}$ and $\rho(z, y)=\theta \Longrightarrow z=y$;

$\left(D_{2}\right) \rho(z, y)=\rho(y, z)$ for all $z, y \in \mathcal{Z}$;

$\left(D_{3}\right) \rho(z, y) \preccurlyeq \rho(z, x)+\rho(x, y)$ for all $z, y, x \in \mathcal{Z}$.

Then $\rho$ is called a dislocated cone metric on $\mathcal{Z}$, and $(\mathcal{Z}, \rho)$ is called a dislocated cone metric space over Banach algebra $\mathcal{A}$. 
Remark 2.3. In a dislocated cone metric space $(\mathcal{Z}, \rho), \rho(z, z)$ need not be zero for $z \in \mathcal{Z}$. Hence every cone metric space over Banach algebras is a dislocated cone metric space over Banach algebras, but the converse is not necessarily true. (see [6]).

Example 2.4. ([6]) Let $\mathcal{A}=\left\{b=\left(b_{i, j}\right)_{3 \times 3}: b_{i, j} \in \mathbb{R}, 1 \leq i, j \leq 3\right\},\|b\|=$ $\sum_{1<i, j \leq 3}\left|b_{i, j}\right|, \mathcal{K}=\left\{b \in \mathcal{A}: b_{i, j} \geq 0,1 \leq i, j \leq 3\right\}$ be a cone in $\overline{\mathcal{A}}$. Let $\overline{\mathcal{Z}}=\mathbb{R}^{+} \cup\{0\}$. Let a mapping $\rho: \mathcal{Z} \times \mathcal{Z} \rightarrow \mathcal{A}$ be define by

$$
\rho(z, y)=\left(\begin{array}{ccc}
z+y & z+y & z+y \\
2 z+2 y & 2 z+2 y & 2 z+2 y \\
3 z+3 y & 3 z+3 y & 3 z+3 y
\end{array}\right), \text { for all } z, y \in \mathcal{Z}
$$

Then $(\mathcal{Z}, \rho)$ is a dislocated cone metric space over a Banach algebra $\mathcal{A}$ but not a cone metric space over a Banach algebra $\mathcal{A}$ since

$$
\rho\left(\frac{1}{2}, \frac{1}{2}\right)=\left(\begin{array}{lll}
1 & 1 & 1 \\
2 & 2 & 2 \\
3 & 3 & 3
\end{array}\right) \neq \theta
$$

Definition 2.5. ([6]) Let $(\mathcal{Z}, \rho)$ be a dislocated cone metric space over Banach algebra $\mathcal{A}, z \in \mathcal{Z}$ and $\left\{z_{i}\right\}$ be a sequence in $(\mathcal{Z}, \rho)$. Then

1. $\left\{z_{i}\right\}$ converges to $z$ whenever for each $c \in \mathcal{A}$ with $\theta \ll c$, there is a natural number $N$ such that $\rho\left(z_{i}, z\right) \ll c$ for all $i \geq N$. We denote this by $z_{i} \rightarrow z(i \rightarrow$ $\infty)$.

2. $\left\{z_{i}\right\}$ is a Cauchy sequence whenever for each $c \in \mathcal{A}$ with $\theta \ll c$, there is a natural number $N$ such that $\rho\left(z_{i}, z_{j}\right) \ll c$ for all $i, j \geq N$.

3. $(\mathcal{Z}, \rho)$ is said to be complete if every Cauchy sequence in $\mathcal{Z}$ is convergent.

Definition 2.6. ([12]) Let $\mathcal{K}$ be a solid cone in a Banach algebra $\mathcal{A}$. A sequence $\left\{z_{i}\right\} \subset \mathcal{K}$ is said to be a $c$-sequence if for each $\theta \ll c$, there exists $N \in \mathbb{N}$ such that $z_{i} \ll c$ for all $i>N$.

Lemma 2.7. ([18]) Let $\mathcal{A}$ be a Banach algebra with a unit $e$ and $\tau \in \mathcal{A}$, then $\lim _{n \rightarrow \infty}\left\|\tau^{n}\right\|^{\frac{1}{n}}$ exists and the spectral radius $\delta(\tau)$ satisfies

$$
\delta(\tau)=\lim _{n \rightarrow \infty}\left\|\tau^{n}\right\|^{\frac{1}{n}}=\inf \left\|\tau^{n}\right\|^{\frac{1}{n}} .
$$

If $\delta(\tau)<1$, then $(e-\tau)$ is invertible in $\mathcal{A}$. Moreover,

$$
(e-\tau)^{-1}=\sum_{k=0}^{\infty} \tau^{k}
$$

and

$$
\delta\left[(e-\tau)^{-1}\right] \leq \frac{1}{1-\delta(\tau)}
$$


Remark 2.8. ([20]). If $\delta(\tau)<1$ then $\left\|\tau^{i}\right\| \rightarrow 0(i \rightarrow \infty)$.

Lemma 2.9. ([7]) If $E$ is a real Banach space with a solid cone $\mathcal{K}$ and $\left\{z_{i}\right\} \subset \mathcal{K}$ be a sequence with $\left\|z_{i}\right\| \rightarrow 0(i \rightarrow \infty)$, then for each $\theta \ll c$, there exists $N \in \mathbb{N}$ such that for any $i>N$, we have $z_{i} \ll c$.

Lemma 2.10. ([6]) Let $(\mathcal{Z}, \rho)$ be a complete dislocated cone metric space over Banach algebra $\mathcal{A}$ and $\mathcal{K}$ be the underlying solid cone. Let $\left\{z_{i}\right\}$ be a sequence in $(\mathcal{Z}, \rho)$. If $\left\{z_{i}\right\}$ converges to $z \in \mathcal{Z}$, then

1. $\left\{\rho\left(z_{i}, z\right)\right\}$ is a c-sequence.

2. For any $j \in \mathbb{N},\left\{\rho\left(z_{i}, z_{i+j}\right)\right\}$ is a c-sequence.

Lemma 2.11. ([12]) Let $\mathcal{A}$ be a real Banach algebra with a solid cone $\mathcal{K}$ and let $\left\{\alpha_{n}\right\}$ and $\left\{\beta_{n}\right\}$ be sequences in $\mathcal{K}$. If $\left\{\alpha_{n}\right\}$ and $\left\{\beta_{n}\right\}$ are $c$-sequences and $k_{1}, k_{2} \in \mathcal{K}$ then $\left\{k_{1} \alpha_{n}+k_{2} \beta_{n}\right\}$ is also a c-sequence.

Lemma 2.12. ([12]) If $E$ is a real Banach space with a solid cone $\mathcal{K}$

1. If $a, b, c \in E$ and $a \preccurlyeq b \ll c$, then $a \ll c$.

2. If $a \in \mathcal{K}$ and $\theta \preccurlyeq a \ll c$ for each $\theta \ll c$, then $a=\theta$.

3. If $a \preccurlyeq \tau a$, where $a, \tau \in \mathcal{K}$ and $\delta(\tau)<1$, then $a=\theta$.

\section{Main results}

First, we introduce the notion of expansive mapping in the setting of dislocated cone metric space over Banach algebra $\mathcal{A}$.

Definition 3.1. Let $(\mathcal{Z}, \rho)$ be a dislocated cone metric space over Banach algebra $\mathcal{A}, \mathcal{K}$ be the underlying solid cone. Then $\mathfrak{F}: \mathcal{Z} \rightarrow \mathcal{Z}$ is called an expansive mapping if there exist $\vartheta, \vartheta^{-1} \in \mathcal{K}$ such that $\delta\left(\vartheta^{-1}\right)<1$ and

$$
\rho(\mathfrak{F} z, \mathfrak{F} y) \succcurlyeq \vartheta \rho(z, y), \text { for all } z, y \in \mathcal{Z} .
$$

Example 3.2. Let $\mathcal{A}=C_{\mathbb{R}}^{1}[0,1]$ and define a norm on $\mathcal{A}$ by $\|z\|=\|z\|_{\infty}+\left\|z^{\prime}\right\|_{\infty}$ for $z \in \mathcal{A}$, where multiplication in $\mathcal{A}$ is defined in the usual way. Then $\mathcal{A}$ is a Banach algebra with unit element $e=1$ and the set $\mathcal{K}=\{z \in \mathcal{A}: z(t) \geq 0, t \in[0,1]\}$ is a cone in $\mathcal{A}$. Let $\mathcal{Z}=[0, \infty)$. Consider a mapping $\rho: \mathcal{Z} \times \mathcal{Z} \rightarrow \mathcal{A}$ define by

$$
\rho(z, y)(t)=(z+y) e^{t}, \text { for all } z, y \in \mathcal{Z} .
$$

Then $(\mathcal{Z}, \rho)$ is a dislocated cone metric space over Banach algebra $\mathcal{A}$. Define a mapping $\mathfrak{F}: \mathcal{Z} \rightarrow \mathcal{Z}$ by $\mathfrak{F} z=2 z$, for all $z \in \mathcal{Z}$. Take $\vartheta=2$. Hence, $\mathfrak{F}$ is expansive mapping. 
Next, we prove the existence of fixed point for generalized expansive mapping in dislocated cone metric space over Banach algebra $\mathcal{A}$ without the assumption of normality of cone.

Theorem 3.3. Let $(\mathcal{Z}, \rho)$ be a complete dislocated cone metric space over Banach algebra $\mathcal{A}$ with a unit $e, \mathcal{K}$ be the underlying solid cone. Let the mapping $\mathfrak{F}: \mathcal{Z} \rightarrow \mathcal{Z}$ be a surjection and satisfy the generalized expansive condition:

$$
\rho(\mathfrak{F} z, \mathfrak{F} y)+\vartheta_{1}[\rho(z, \mathfrak{F} y)+\rho(y, \mathfrak{F} z)] \succcurlyeq \vartheta_{2} \rho(z, y)+\vartheta_{3} \rho(z, \mathfrak{F} z)+\vartheta_{4} \rho(y, \mathfrak{F} y),
$$

for all $z, y \in \mathcal{Z}$, where $\vartheta_{j} \in \mathcal{K}(j=1,2,3,4)$ such that $\left(\vartheta_{2}+\vartheta_{3}-3 \vartheta_{1}\right)^{-1},\left(\vartheta_{2}-\vartheta_{1}+\right.$ $\left.\vartheta_{4}\right)^{-1} \in \mathcal{K}$ and spectral radius $\delta\left[\left(\vartheta_{2}+\vartheta_{3}-3 \vartheta_{1}\right)^{-1}\left(e+\vartheta_{1}-\vartheta_{4}\right)\right]<1$. Then $\mathfrak{F}$ has a fixed point $z_{*}$ in $\mathcal{Z}$.

Proof. Let $z_{0} \in \mathcal{Z}$. Since $\mathfrak{F}$ is surjective, there exists $z_{1} \in \mathcal{Z}$ such that $\mathfrak{F} z_{1}=z_{0}$. Again, we can choose $z_{2} \in \mathcal{Z}$ such that $\mathfrak{F} z_{2}=z_{1}$. Continuing this process, we can construct a sequence $\left\{z_{i}\right\}$ in $(\mathcal{Z}, \rho)$ by

$$
z_{i}=\mathfrak{F} z_{i+1}, \text { for } i=0,1,2, \ldots
$$

Suppose $z_{k}=z_{k+1}$ for some $k \in \mathbb{N}$, then $z_{*}=z_{k}$ is a fixed point of $\mathfrak{F}$ and the result is proved. Hence, we assume that $z_{i+1} \neq z_{i}, \forall i \in \mathbb{N}$. Using (3.2) and (3.3), we get

$$
\begin{aligned}
\rho\left(\mathfrak{F} z_{i+1}, \mathfrak{F} z_{i}\right)+\vartheta_{1}\left[\rho\left(z_{i+1}, \mathfrak{F} z_{i}\right)+\rho\left(z_{i}, \mathfrak{F} z_{i+1}\right)\right] \succcurlyeq \vartheta_{2} \rho\left(z_{i+1}, z_{i}\right) & \\
& +\vartheta_{3} \rho\left(z_{i+1}, \mathfrak{F} z_{i+1}\right)+\vartheta_{4} \rho\left(z_{i}, \mathfrak{F} z_{i}\right) \\
\rho\left(z_{i}, z_{i-1}\right)+\vartheta_{1}\left[\rho\left(z_{i+1}, z_{i-1}\right)+\rho\left(z_{i}, z_{i}\right)\right] & \succcurlyeq \vartheta_{2} \rho\left(z_{i+1}, z_{i}\right)+\vartheta_{3} \rho\left(z_{i+1}, z_{i}\right)+\vartheta_{4} \rho\left(z_{i}, z_{i-1}\right) \\
\rho\left(z_{i}, z_{i-1}\right)+\vartheta_{1}\left[3 \rho\left(z_{i+1}, z_{i}\right)+\rho\left(z_{i}, z_{i-1}\right)\right] & \succcurlyeq\left(\vartheta_{2}+\vartheta_{3}\right) \rho\left(z_{i+1}, z_{i}\right)+\vartheta_{4} \rho\left(z_{i}, z_{i-1}\right) \\
\left(e+\vartheta_{1}-\vartheta_{4}\right) \rho\left(z_{i}, z_{i-1}\right) & \succcurlyeq\left(\vartheta_{2}+\vartheta_{3}-3 \vartheta_{1}\right) \rho\left(z_{i+1}, z_{i}\right) \\
\left(\vartheta_{2}+\vartheta_{3}-3 \vartheta_{1}\right) \rho\left(z_{i+1}, z_{i}\right) & \preccurlyeq\left(e+\vartheta_{1}-\vartheta_{4}\right) \rho\left(z_{i}, z_{i-1}\right) \\
\rho\left(z_{i}, z_{i+1}\right) & \preccurlyeq \tau \rho\left(z_{i-1}, z_{i}\right),
\end{aligned}
$$

where $\tau=\left(\vartheta_{2}+\vartheta_{3}-3 \vartheta_{1}\right)^{-1}\left(e+\vartheta_{1}-\vartheta_{4}\right)$.

Hence, from (3.4), we get

$$
\begin{aligned}
\rho\left(z_{i}, z_{i+1}\right) & \preccurlyeq \tau \rho\left(z_{i-1}, z_{i}\right) \\
& \preccurlyeq \tau^{2} \rho\left(z_{i-2}, z_{i-1}\right) \\
& \vdots \\
\rho\left(z_{i}, z_{i+1}\right) & \preccurlyeq \tau^{i} \rho\left(z_{0}, z_{1}\right), \text { for all } i \in \mathbb{N} .
\end{aligned}
$$

Since $\delta(\tau)<1$, it follows, by Lemma 2.7, that $(e-\tau)$ is invertible in $\mathcal{A}$. Moreover,

$$
(e-\tau)^{-1}=\sum_{k=0}^{\infty} \tau^{k} .
$$


Also, by Remark 2.8, we get

$$
\left\|\tau^{i}\right\| \rightarrow 0(i \rightarrow \infty)
$$

Hence, for $i, j \in \mathbb{N}$ with $i<j$, using (3.5) and (3.6), we have

$$
\begin{aligned}
\rho\left(z_{i}, z_{j}\right) \preccurlyeq & \rho\left(z_{i}, z_{i+1}\right)+\rho\left(z_{i+1}, z_{j}\right) \\
\preccurlyeq & \rho\left(z_{i}, z_{i+1}\right)+\rho\left(z_{i+1}, z_{i+2}\right)+\rho\left(z_{i+2}, z_{j}\right) \\
\preccurlyeq & \rho\left(z_{i}, z_{i+1}\right)+\rho\left(z_{i+1}, z_{i+2}\right)+\rho\left(z_{i+2}, z_{i+3}\right) \\
& +\cdots+\rho\left(z_{j-2}, z_{j-1}\right)+\rho\left(z_{j-1}, z_{j}\right) \\
\preccurlyeq & \tau^{i} \rho\left(z_{0}, z_{1}\right)+\tau^{i+1} \rho\left(z_{0}, z_{1}\right)+\tau^{i+2} \rho\left(z_{0}, z_{1}\right) \\
& +\cdots+\tau^{j-2} \rho\left(z_{0}, z_{1}\right)+\tau^{j-1} \rho\left(z_{0}, z_{1}\right) \\
= & \tau^{i}\left(e+\tau+\tau^{2}+\cdots+\tau^{j-i-2}+\tau^{j-i-1}\right) \rho\left(z_{0}, z_{1}\right) \\
\preccurlyeq & \tau^{i}\left(\sum_{k=0}^{\infty} \tau^{k}\right) \rho\left(z_{0}, z_{1}\right) \\
= & \tau^{i}(e-\tau)^{-1} \rho\left(z_{0}, z_{1}\right) .
\end{aligned}
$$

Therefore, using (3.7), we have that $\left\|\tau^{i}(e-\tau)^{-1} \rho\left(z_{0}, z_{1}\right)\right\| \rightarrow 0(i \rightarrow \infty)$, and it follows, by Lemma 2.9 , that for any $c \in \mathcal{A}$ with $\theta \ll c$, there exists $N \in \mathbb{N}$ such that

$$
\rho\left(z_{i}, z_{j}\right) \preccurlyeq \tau^{i}(e-\tau)^{-1} \rho\left(z_{0}, z_{1}\right) \ll c \text {, for all } j>i>N,
$$

which implies, by Lemma 2.12 and Definition 2.5, that $\left\{z_{i}\right\}$ is a Cauchy sequence. Since $(\mathcal{Z}, \rho)$ is complete, there exists $z_{*} \in \mathcal{Z}$ such that $z_{i} \rightarrow z_{*}(i \rightarrow \infty)$. Since $\mathfrak{F}$ is a surjection mapping, there exists a point $y_{*}$ in $\mathcal{Z}$ such that $\mathfrak{F} y_{*}=z_{*}$. Next, we show that $y_{*}=z_{*}$. Using (3.2) and (3.3), we have that

$$
\begin{aligned}
\rho\left(z_{i}, z_{*}\right)= & \rho\left(\mathfrak{F} z_{i+1}, \mathfrak{F} y_{*}\right) \\
\succcurlyeq & -\vartheta_{1}\left[\rho\left(z_{i+1}, \mathfrak{F} y_{*}\right)+\rho\left(y_{*}, \mathfrak{F} z_{i+1}\right)\right]+\vartheta_{2} \rho\left(z_{i+1}, y_{*}\right) \\
& +\vartheta_{3} \rho\left(z_{i+1}, \mathfrak{F} z_{i+1}\right)+\vartheta_{4} \rho\left(y_{*}, \mathfrak{F} y_{*}\right) \\
\succcurlyeq & -\vartheta_{1}\left[\rho\left(z_{i+1}, z_{*}\right)+\rho\left(y_{*}, z_{i}\right)\right]+\vartheta_{2} \rho\left(z_{i+1}, y_{*}\right) \\
& +\vartheta_{3} \rho\left(z_{i+1}, z_{i}\right)+\vartheta_{4} \rho\left(y_{*}, z_{*}\right) \\
\rho\left(z_{i}, z_{i+1}\right)+\rho\left(z_{i+1}, z_{*}\right) \succcurlyeq & -\vartheta_{1} \rho\left(z_{i+1}, z_{*}\right)-\vartheta_{1}\left[\rho\left(y_{*}, z_{i+1}\right)-\rho\left(z_{i}, z_{i+1}\right)\right] \\
& +\vartheta_{2} \rho\left(z_{i+1}, y_{*}\right)+\vartheta_{3} \rho\left(z_{i+1}, z_{i}\right) \\
& +\vartheta_{4}\left[\rho\left(y_{*}, z_{i+1}\right)-\rho\left(z_{*}, z_{i+1}\right)\right] \\
\left(\vartheta_{2}-\vartheta_{1}+\vartheta_{4}\right) \rho\left(z_{i+1}, y_{*}\right) \preccurlyeq & \left(e+\vartheta_{1}+\vartheta_{4}\right) \rho\left(z_{i+1}, z_{*}\right)+\left(e-\vartheta_{1}-\vartheta_{3}\right) \rho\left(z_{i}, z_{i+1}\right) \\
\rho\left(z_{i+1}, y_{*}\right) \preccurlyeq & \left(\vartheta_{2}-\vartheta_{1}+\vartheta_{4}\right)-1\left[\left(e+\vartheta_{1}+\vartheta_{4}\right) \rho\left(z_{i+1}, z_{*}\right)\right. \\
& \left.+\left(e-\vartheta_{1}-\vartheta_{3}\right) \rho\left(z_{i}, z_{i+1}\right)\right] .
\end{aligned}
$$

This implies that

$$
\rho\left(z_{i+1}, y_{*}\right) \preccurlyeq \alpha_{1} \rho\left(z_{i+1}, z_{*}\right)+\alpha_{2} \rho\left(z_{i}, z_{i+1}\right),
$$


where $\alpha_{1}=\left(\vartheta_{2}-\vartheta_{1}+\vartheta_{4}\right)^{-1}\left(e+\vartheta_{1}+\vartheta_{4}\right), \alpha_{2}=\left(\vartheta_{2}-\vartheta_{1}+\vartheta_{4}\right)^{-1}\left(e-\vartheta_{1}-\vartheta_{3}\right) \in \mathcal{K}$. Now, by Lemma 2.10, Lemma 2.11; $\left\{\rho\left(z_{i+1}, z_{*}\right)\right\},\left\{\rho\left(z_{i}, z_{i+1}\right)\right\}$ and $\left\{\alpha_{1} \rho\left(z_{i+1}, z_{*}\right)+\right.$ $\left.\alpha_{2} \rho\left(z_{i}, z_{i+1}\right)\right\}$ are $c$-sequences. Hence, for any $c \in \mathcal{A}$ with $\theta \ll c$, there exists $N \in \mathbb{N}$ such that

$$
\rho\left(z_{i+1}, y_{*}\right) \preccurlyeq \alpha_{1} \rho\left(z_{i+1}, z_{*}\right)+\alpha_{2} \rho\left(z_{i}, z_{i+1}\right) \ll c \text {, for all } i>N \text {, }
$$

which implies that $z_{i+1} \rightarrow y_{*}$. Since the limit of a convergent sequence in cone metric space is unique, we have that $y_{*}=z_{*}$. Hence, $z_{*}$ is a fixed point of $\mathfrak{F}$.

Remark 3.4. Note that $\mathfrak{F}$ may have more than one fixed point (e.g. see $[11,1]$ ).

Theorem 3.5. Let $(\mathcal{Z}, \rho)$ be a complete dislocated cone metric space over Banach algebra $\mathcal{A}$ with a unit $e, \mathcal{K}$ be the underlying solid cone. Let the mapping $\mathfrak{F}: \mathcal{Z} \rightarrow \mathcal{Z}$ be a surjection and satisfy the following condition:

$$
\rho(\mathfrak{F} z, \mathfrak{F} y) \succcurlyeq \vartheta_{1} \rho(z, y)+\vartheta_{2} \rho(z, \mathfrak{F} y) \text {, for all } z, y \in \mathcal{Z} \text {, }
$$

where $\vartheta_{1}, \vartheta_{2} \in \mathcal{K}$ such that $\left(\vartheta_{1}+\vartheta_{2}\right)^{-1} \in \mathcal{K}$ and spectral radius $\delta\left[\left(\vartheta_{1}+\vartheta_{2}\right)^{-1}\left(e+\vartheta_{2}\right)\right]<1$. Then $\mathfrak{F}$ has a fixed point $z_{*}$ in $\mathcal{Z}$.

Proof. Let $z_{0}$ be an arbitrary point in $\mathcal{Z}$. Since $\mathfrak{F}$ is surjective, there exists $z_{1} \in \mathcal{Z}$ such that $\mathfrak{F} z_{1}=z_{0}$. Again, we can choose $z_{2} \in \mathcal{Z}$ such that $\mathfrak{F} z_{2}=z_{1}$. Continuing this process, we can construct a sequence $\left\{z_{i}\right\}$ in $(\mathcal{Z}, \rho)$ by

$$
z_{i}=\mathfrak{F} z_{i+1}, \text { for } i=0,1,2, \ldots
$$

Suppose $z_{j-1}=z_{j}$ for some $j \in \mathbb{N}$, then $z_{*}=z_{j}$ is a fixed point of $\mathfrak{F}$ and the result is proved. Hence, we assume that $z_{i} \neq z_{i-1}$ for all $i \in \mathbb{N}$. Now, using (3.8) and (3.9), we have

$$
\begin{aligned}
\rho\left(z_{i}, z_{i-1}\right) & =\rho\left(\mathfrak{F} z_{i+1}, \mathfrak{F} z_{i}\right) \\
& \succcurlyeq \vartheta_{1} \rho\left(z_{i+1}, z_{i}\right)+\vartheta_{2} \rho\left(z_{i+1}, z_{i-1}\right) \\
& \succcurlyeq \vartheta_{1} \rho\left(z_{i+1}, z_{i}\right)+\vartheta_{2}\left[\rho\left(z_{i+1}, z_{i}\right)-\rho\left(z_{i-1}, z_{i}\right)\right] \\
\left(e+\vartheta_{2}\right) \rho\left(z_{i}, z_{i-1}\right) & \succcurlyeq\left(\vartheta_{1}+\vartheta_{2}\right) \rho\left(z_{i+1}, z_{i}\right) \\
\rho\left(z_{i}, z_{i+1}\right) & \preccurlyeq\left(\vartheta_{1}+\vartheta_{2}\right)^{-1}\left(e+\vartheta_{2}\right) \rho\left(z_{i-1}, z_{i}\right) \\
\rho\left(z_{i}, z_{i+1}\right) & \preccurlyeq \tau \rho\left(z_{i-1}, z_{i}\right),
\end{aligned}
$$

where $\tau=\left(\vartheta_{1}+\vartheta_{2}\right)^{-1}\left(e+\vartheta_{2}\right)$.

Hence, from (3.10), we have

$$
\begin{aligned}
\rho\left(z_{i}, z_{i+1}\right) & \preccurlyeq \tau \rho\left(z_{i-1}, z_{i}\right) \\
& \preccurlyeq \tau^{2} \rho\left(z_{i-2}, z_{i-1}\right) \\
& \vdots \\
\rho\left(z_{i}, z_{i+1}\right) & \preccurlyeq \tau^{i} \rho\left(z_{0}, z_{1}\right), \text { for all } i \in \mathbb{N} .
\end{aligned}
$$


Using the same argument to the proof in Theorem 3.3, we get that $\left\{z_{i}\right\}$ is a Cauchy sequence. Since $(\mathcal{Z}, \rho)$ is complete, there exists $z_{*} \in \mathcal{Z}$ such that $z_{i} \rightarrow z_{*}(i \rightarrow \infty)$. Since $\mathfrak{F}$ is a surjection mapping, there exists a point $z_{* *}$ in $\mathcal{Z}$ such that $\mathfrak{F} z_{* *}=z_{*}$. Now, we show that $z_{* *}=z_{*}$. Using (3.8) and (3.9), we have that

$$
\begin{aligned}
\rho\left(z_{*}, z_{i}\right) & =\rho\left(\mathfrak{F} z_{* *}, \mathfrak{F} z_{i+1}\right) \\
& \succcurlyeq \vartheta_{1} \rho\left(z_{* *}, z_{i+1}\right)+\vartheta_{2} \rho\left(z_{* *}, \mathfrak{F} z_{i+1}\right) \\
& =\vartheta_{1} \rho\left(z_{* *}, z_{i+1}\right)+\vartheta_{2} \rho\left(z_{* *}, z_{i}\right) \\
\rho\left(z_{*}, z_{i+1}\right)+\rho\left(z_{i+1}, z_{i}\right) & \succcurlyeq \vartheta_{1} \rho\left(z_{* *}, z_{i+1}\right)+\vartheta_{2}\left[\rho\left(z_{* *}, z_{i+1}\right)-\rho\left(z_{i}, z_{i+1}\right)\right] \\
\left(\vartheta_{1}+\vartheta_{2}\right) \rho\left(z_{i+1}, z_{* *}\right) & \preccurlyeq \rho\left(z_{i+1}, z_{*}\right)+\left(e+\vartheta_{2}\right) \rho\left(z_{i}, z_{i+1}\right) \\
\rho\left(z_{i+1}, z_{* *}\right) & \preccurlyeq\left(\vartheta_{1}+\vartheta_{2}\right)^{-1}\left[\rho\left(z_{i+1}, z_{*}\right)+\left(e+\vartheta_{2}\right) \rho\left(z_{i}, z_{i+1}\right)\right] .
\end{aligned}
$$

This implies that

$$
\rho\left(z_{i+1}, z_{* *}\right) \preccurlyeq \beta_{1} \rho\left(z_{i+1}, z_{*}\right)+\beta_{2} \rho\left(z_{i}, z_{i+1}\right),
$$

where $\beta_{1}=\left(\vartheta_{1}+\vartheta_{2}\right)^{-1}, \beta_{2}=\left(\vartheta_{1}+\vartheta_{2}\right)^{-1}\left(e+\vartheta_{2}\right) \in \mathcal{K}$. Now, by Lemma 2.10, Lemma $2.11 ;\left\{\rho\left(z_{i+1}, z_{*}\right)\right\},\left\{\rho\left(z_{i}, z_{i+1}\right)\right\}$ and $\left\{\beta_{1} \rho\left(z_{i+1}, z_{*}\right)+\beta_{2} \rho\left(z_{i}, z_{i+1}\right)\right\}$ are $c$-sequences. Hence, for any $c \in \mathcal{A}$ with $\theta \ll c$, there exists $N \in \mathbb{N}$ such that

$$
\rho\left(z_{i+1}, z_{* *}\right) \preccurlyeq \beta_{1} \rho\left(z_{i+1}, z_{*}\right)+\beta_{2} \rho\left(z_{i}, z_{i+1}\right) \ll c, \text { for all } i>N \text {, }
$$

which implies that $z_{i+1} \rightarrow z_{* *}$. Since the limit of a convergent sequence in a cone metric space is unique, we have that $z_{* *}=z_{*}$. Hence, $z_{*}$ is a fixed point of $\mathfrak{F}$.

Corollary 3.6. Let $(\mathcal{Z}, \rho)$ be a complete dislocated cone metric space over Banach algebra $\mathcal{A}$ with a unit $e, \mathcal{K}$ be the underlying solid cone. Let the mapping $\mathfrak{F}: \mathcal{Z} \rightarrow \mathcal{Z}$ be a surjection and satisfy the following condition:

$$
\rho(\mathfrak{F} z, \mathfrak{F} y) \succcurlyeq \vartheta_{1} \rho(z, y)+\vartheta_{2} \rho(z, \mathfrak{F} z)+\vartheta_{3} \rho(y, \mathfrak{F} y)
$$

for all $z, y \in \mathcal{Z}$. where $\vartheta_{k} \in \mathcal{K}(k=1,2,3)$ such that $\left(\vartheta_{1}+\vartheta_{2}\right)^{-1},\left(\vartheta_{1}+\vartheta_{3}\right)^{-1} \in \mathcal{K}$ and spectral radius $\delta\left[\left(\vartheta_{1}+\vartheta_{2}\right)^{-1}\left(e-\vartheta_{3}\right)\right]<1$. Then $\mathfrak{F}$ has a fixed point $z_{*}$ in $\mathcal{Z}$.

Proof. Putting $\vartheta_{1}=\theta$ in Theorem 3.3, the result follows.

Corollary 3.7. Let $(\mathcal{Z}, \rho)$ be a complete dislocated cone metric space over Banach algebra $\mathcal{A}$ with a unit $e, \mathcal{K}$ be the underlying solid cone. Let the mapping $\mathfrak{F}: \mathcal{Z} \rightarrow \mathcal{Z}$ be a surjection and satisfy the following condition:

$$
\rho(\mathfrak{F} z, \mathfrak{F} y) \succcurlyeq \vartheta_{1} \rho(z, \mathfrak{F} z)+\vartheta_{2} \rho(y, \mathfrak{F} y),
$$

for all $z, y \in \mathcal{Z}$. where $\vartheta_{k} \in \mathcal{K}(k=1,2)$ such that $\vartheta_{1}{ }^{-1}, \vartheta_{2}{ }^{-1} \in \mathcal{K}$ and spectral radius $\delta\left[\vartheta_{1}^{-1}\left(e-\vartheta_{2}\right)\right]<1$. Then $\mathfrak{F}$ has a fixed point $z_{*}$ in $\mathcal{Z}$.

Proof. Putting $\vartheta_{1}=\vartheta_{2}=\theta$ in Theorem 3.3, the result follows. 
Theorem 3.8. Let $(\mathcal{Z}, \rho)$ be a complete dislocated cone metric space over Banach algebra $\mathcal{A}$ with a unit e, $\mathcal{K}$ be the underlying solid cone. Let the mapping $\mathfrak{F}: \mathcal{Z} \rightarrow \mathcal{Z}$ be a surjection and satisfy the following condition:

$$
\rho(\mathfrak{F} z, \mathfrak{F} y) \succcurlyeq \vartheta \rho(z, y),
$$

for all $z, y \in \mathcal{Z}$. where $\vartheta, \vartheta^{-1} \in \mathcal{K}$ such that spectral radius $\delta\left(\vartheta^{-1}\right)<1$. Then $\mathfrak{F}$ has a unique fixed point $z_{*}$ in $\mathcal{Z}$.

Proof. Using Theorem 3.3, Theorem 3.5, we only need to show that the fixed point is unique. Suppose that $y_{*}$ is another fixed point of $\mathfrak{F}$, then using (3.14), we have that

$$
\begin{aligned}
\rho\left(z_{*}, y_{*}\right) & =\rho\left(\mathfrak{F} z_{*}, \mathfrak{F} y_{*}\right) \\
& \succcurlyeq \vartheta \rho\left(z_{*}, y_{*}\right) \\
\rho\left(z_{*}, y_{*}\right) & \preccurlyeq \vartheta^{-1} \rho\left(z_{*}, y_{*}\right)=\tau \rho\left(z_{*}, y_{*}\right),
\end{aligned}
$$

where $\tau=\vartheta^{-1}$.

Hence, we have

$$
\begin{aligned}
& \rho\left(z_{*}, y_{*}\right) \preccurlyeq \tau \rho\left(z_{*}, y_{*}\right) \\
& \preccurlyeq \tau^{2} \rho\left(z_{*}, y_{*}\right) \\
& \vdots \\
& \rho\left(z_{*}, y_{*}\right) \preccurlyeq \tau^{i} \rho\left(z_{*}, y_{*}\right), \text { for all } i \in \mathbb{N} .
\end{aligned}
$$

Since $\delta(\tau)<1$, then, by Remark 2.8 , it follows that

$$
\left\|\tau^{i}\right\| \rightarrow 0(i \rightarrow \infty)
$$

Hence, we have that $\left\|\tau^{i} \rho\left(z_{*}, y_{*}\right)\right\| \rightarrow 0(i \rightarrow \infty)$ and by Lemma 2.9, it follows that for any $c \in \mathcal{A}$ with $\theta \ll c$, there exists $N \in \mathbb{N}$ such that

$$
\rho\left(z_{*}, y_{*}\right) \preccurlyeq \tau^{i} \rho\left(z_{*}, y_{*}\right) \ll c, \text { for all } i>N,
$$

which implies that $\rho\left(z_{*}, y_{*}\right)=\theta$. Therefore $z_{*}=y_{*}$. This completes the proof.

Corollary 3.9. Let $(\mathcal{Z}, \rho)$ be a complete dislocated cone metric space over Banach algebra $\mathcal{A}$ with a unit $e, \mathcal{K}$ be the underlying solid cone. Let the mapping $\mathfrak{F}: \mathcal{Z} \rightarrow \mathcal{Z}$ be a surjection and satisfy the following condition:

$$
\rho\left(\mathfrak{F}^{m} z, \mathfrak{F}^{m} y\right) \succcurlyeq \vartheta \rho(z, y), \quad m \in \mathbb{Z}^{+}
$$

for all $z, y \in \mathcal{Z}$. where $\vartheta, \vartheta^{-1} \in \mathcal{K}$ such that $\delta\left(\vartheta^{-1}\right)<1$. Then $\mathfrak{F}$ has a unique fixed point $z_{*}$ in $\mathcal{Z}$.

Proof. Using Theorem 3.8, we get that $\mathfrak{F}^{m}$ a has a fixed point $z$ in $\mathcal{Z}$. Since $\mathfrak{F}^{m}(\mathfrak{F} z)=$ $\mathfrak{F}\left(\mathfrak{F}^{m} z\right)=\mathfrak{F} z$, then $\mathfrak{F} z$ is also a fixed point of $\mathfrak{F}^{m}$. Thus $\mathfrak{F} z=z, z$ is a fixed of $\mathfrak{F}$. Since the fixed in Theorem 3.8 is unique, the result follows. 
Theorem 3.10. Let $(\mathcal{Z}, \rho)$ be a complete dislocated cone metric space over Banach algebra $\mathcal{A}$ with a unit e, $\mathcal{K}$ be the underlying solid cone. Let the mapping $\mathfrak{F}: \mathcal{Z} \rightarrow \mathcal{Z}$ be a continuous, surjection and satisfy the following condition:

$$
\rho(\mathfrak{F} z, \mathfrak{F} y) \succcurlyeq \vartheta\{\rho(z, y), \rho(z, \mathfrak{F} z), \rho(y, \mathfrak{F} y)\},
$$

for all $z, y \in \mathcal{Z}$, where $\vartheta_{j} \in \mathcal{K}(j=1,2,3,4)$ such that $\vartheta, \vartheta^{-1} \in \mathcal{K}$ and spectral radius $\delta\left(\vartheta^{-1}\right)<1$. Then $\mathfrak{F}$ has a fixed point $z_{*}$ in $\mathcal{Z}$.

Proof. Let $z_{0}$ be an arbitrary point in $\mathcal{Z}$. Since $\mathfrak{F}$ is surjective, there exists $z_{1} \in \mathcal{Z}$ such that $\mathfrak{F} z_{1}=z_{0}$. Again, we can choose $z_{2} \in \mathcal{Z}$ such that $\mathfrak{F} z_{2}=z_{1}$. Continuing this process, we can construct a sequence $\left\{z_{i}\right\}$ in $\mathcal{Z}$ by

$$
z_{i}=\mathfrak{F} z_{i+1}, \text { for } i=0,1,2, \ldots
$$

Suppose $z_{j-1}=z_{j}$ for some $j \in \mathbb{N}$, then $z_{*}=z_{j-1}$ is a fixed point of $\mathfrak{F}$ and the result is proved. Hence, we assume that $z_{i-1} \neq z_{i}$ for all $i \in \mathbb{N}$. Now, using (3.16) and (3.17), we have

$$
\begin{aligned}
\rho\left(z_{i-1}, z_{i}\right) & =\rho\left(\mathfrak{F} z_{i}, \mathfrak{F} z_{i+1}\right) \\
& \succcurlyeq \vartheta\left\{\rho\left(z_{i}, z_{i+1}\right), \rho\left(z_{i}, \mathfrak{F} z_{i}\right), \rho\left(z_{i+1}, \mathfrak{F} z_{i+1}\right)\right\} \\
& =\vartheta\left\{\rho\left(z_{i}, z_{i+1}\right), \rho\left(z_{i}, z_{i-1}\right)\right\} .
\end{aligned}
$$

We consider the following two cases:

1. If $\rho\left(z_{i-1}, z_{i}\right) \succcurlyeq \vartheta \rho\left(z_{i}, z_{i-1}\right)$ then $\rho\left(z_{i-1}, z_{i}\right) \preccurlyeq \vartheta^{-1} \rho\left(z_{i-1}, z_{i}\right)$. Hence, by Lemma 2.12, $\rho\left(z_{i-1}, z_{i}\right)=\theta$, that is $z_{i-1}=z_{i}$. This is a contradiction.

2. If $\rho\left(z_{i-1}, z_{i}\right) \succcurlyeq \vartheta \rho\left(z_{i}, z_{i+1}\right)$ then $\rho\left(z_{i}, z_{i+1}\right) \preccurlyeq \vartheta^{-1} \rho\left(z_{i-1}, z_{i}\right)=\tau \rho\left(z_{i-1}, z_{i}\right)$.

Hence, we have

$$
\begin{aligned}
\rho\left(z_{i}, z_{i+1}\right) & \preccurlyeq \tau \rho\left(z_{i-1}, z_{i}\right) \\
& \preccurlyeq \tau^{2} \rho\left(z_{i-2}, z_{i-1}\right) \\
& \vdots \\
\rho\left(z_{i}, z_{i+1}\right) & \preccurlyeq \tau^{i} \rho\left(z_{0}, z_{1}\right), \text { for all } i \in \mathbb{N} .
\end{aligned}
$$

Using the same argument to the proof in Theorem 3.3, we get that $\left\{z_{i}\right\}$ is a Cauchy sequence. Since $(\mathcal{Z}, \rho)$ is complete, there exists $z_{*} \in \mathcal{Z}$ such that $z_{i} \rightarrow z_{*}(i \rightarrow \infty)$. To show that $z_{*}$ is a fixed point of $\mathfrak{F}$, since $\mathfrak{F}$ is continuous, so $\mathfrak{F} z_{i} \rightarrow \mathfrak{F} z_{*}(i \rightarrow \infty)$, which implies that $z_{i-1} \rightarrow \mathfrak{F} z_{*}(i \rightarrow \infty)$. Hence, $\mathfrak{F} z_{*}=z_{*}$. This completes the proof.

Example 3.11. Let $\mathcal{A}=C_{\mathbb{R}}^{1}[0,1 / 5]$ and define a norm on $\mathcal{A}$ by $\|z\|=\|z\|_{\infty}+\left\|z^{\prime}\right\|_{\infty}$ for $z \in \mathcal{A}$, where multiplication in $\mathcal{A}$ is defined in the usual way. Then $\mathcal{A}$ is a Banach algebra with unit element $e=1$ and the set $\mathcal{K}=\{z \in \mathcal{A}: z(t) \geq 0, t \in[0,1 / 5]\}$ is a non normal cone in $\mathcal{A}$. Let $\mathcal{Z}=[0, \infty)$. Consider a mapping $\rho: \mathcal{Z} \times \mathcal{Z} \rightarrow \mathcal{A}$ define by

$$
\rho(z, y)(t)=(z+y) e^{t}, \text { for all } z, y \in \mathcal{Z} .
$$


Then $(\mathcal{Z}, \rho)$ is a dislocated cone metric space over Banach algebra $\mathcal{A}$. Define a mapping $\mathfrak{F}: \mathcal{Z} \rightarrow \mathcal{Z}$ by $\mathfrak{F} z=2 z$, for all $z \in \mathcal{Z}$. Let $\vartheta \in \mathcal{K}$ be defined by $\vartheta(t)=\frac{5}{3 t+4}$. Simple calculations show that all the conditions of Theorem 3.8 are satisfied and $z_{*}=\theta$ is the unique fixed point of $\mathfrak{F}$.

\section{Conclusion}

The aim of this paper is to introduce the notion of generalized expansive mappings on dislocated cone metric space over Banach algebras and prove some fixed point theorems for such mappings. Our results are actual generalization of the recent results in $[11,2,10,1,3,19]$ and others in the literature. We hope the results will be useful in fixed point theory and may be generalized in further spaces with efficient conditions.

\section{Competing interests}

The authors declare that they have no competing interests.

\section{Authors contributions}

All authors have equally contributed towards writing this article. All authors read and approved the final manuscript.

\section{References}

[1] C.T. Aage, J.N. Salunke, Some fixed point theorems for expansion onto mappings on cone metric spaces, Acta Mathematica Sinica (English series) 27 (6) (2011) 1101-1106.

[2] A. Auwalu, A note on some fixed point theorems for generalized expansive mappings in cone metric spaces over Banach algebras, AIP Conference Proc. 1997 (020004) (2018) 1-7.

[3] S. Chouhan, N. Malviya, A fixed point theorem for expansive type mappings in cone metric spaces, Int. Math. Forum 6 (18) (2011) 891-897.

[4] Deepmala, R.P. Agarwal, Existence and uniqueness of solutions for certain functional equations and system of functional equations arising in dynamic programming, An. St. Univ. Ovidius Constanta, Math. Series 24 (1) (2016) 3-28.

[5] Deepmala, A.K. Das, On solvability for certain functional equations arising in dynamic programming, Mathematics and Computing, Springer Proceedings in Mathematics and Statistics 139 (2015) 79-94.

[6] R. George, R. Rajagopalan, H.A. Nabwey, S. Radenović, Dislocated cone metric space over Banach algebras and $\alpha$-quasi contraction mappings of Perov type, Fixed Point Theory Appl. (2017) 2017:24. 
[7] H. Huang, S. Radenović, Common fixed point theorems of generalized Lipschitz mappings in cone metric spaces over Banach algebras, Appl. Math. Inf. Sci. 9 (6) (2015) 2983-2990.

[8] H. Huang, S. Radenović, Common fixed point theorems of generalized Lipschitz mappings in cone b-metric spaces over Banach algebras and applications, J. Nonlinear Sci. Appl. 8 (2015) 787-799.

[9] L.G. Huang, X. Zhang, Cone metric spaces and fixed point theorems for contractive mappings, J. Math. Anal. Appl. 332 (2) (2007) 1468-1476.

[10] X. Huang, C. Zhu, X. Wen, Fixed point theorems for expanding mappings in cone metric spaces, Math. Reports 14 (2) (2012) 141-148.

[11] B. Jiang, S. Xu, H. Huang, Z. Cai, Some fixed point theorems for generalized expansive mappings in cone metric spaces over Banach algebras, J. Comput. Anal. Appl. 21 (6) (2016) 1103-1114.

[12] Z. Kadelburg, S. Radenović, A note on various types of cones and fixed point results in cone metric spaces, Asian J. Math. Appl., Article ID ama0104 (2013) 7 pages.

[13] H. Liu, S. Xu, Cone metric spaces with Banach algebras and fixed point theorems of generalized Lipschitz mappings, Fixed Point Theory Appl. (2013) 2013:320.

[14] L.N. Mishra, M. Sen, On the concept of existence and local attractivity of solutions for some quadratic Volterra integral equation of fractional order, Appl. Math. Comput. 285 (2016) 174-183.

[15] L.N. Mishra, M. Sen, R.N. Mohapatra, On existence theorems for some generalized nonlinear functional-integral equations with applications, Filomat 31 (7) (2017) 2081-2091.

[16] V.N. Mishra, Some Problems on Approximations of Functions in Banach Spaces, Ph.D. Thesis, Indian Institute of Technology, Roorkee 247 667, Uttarakhand, India, 2016.

[17] P.P. Murthy, Rashmi, V.N. Mishra, Tripled coincidence point theorem for compatible maps in fuzzy metric spaces, Electronic Journal of Mathematical Analysis and Applications 4 (2) (2016) 96-106.

[18] W. Rudin, Functional Analysis, 2nd edition, McGraw-Hill, New York, 1991.

[19] S.Z. Wang, B.Y. Li, Z.M. Gao, K. Iseki, Some fixed point theorems for expansion mappings, Math. Japon. 29 (1984) 631-636.

[20] S. Xu, S. Radenović, Fixed point theorems of generalized Lipschitz mappings on cone metric spaces over Banach algebras without assumption of normality, Fixed Point Theory Appl. (2014) 2014:102. 
DOI: $10.7862 /$ rf.2019.2

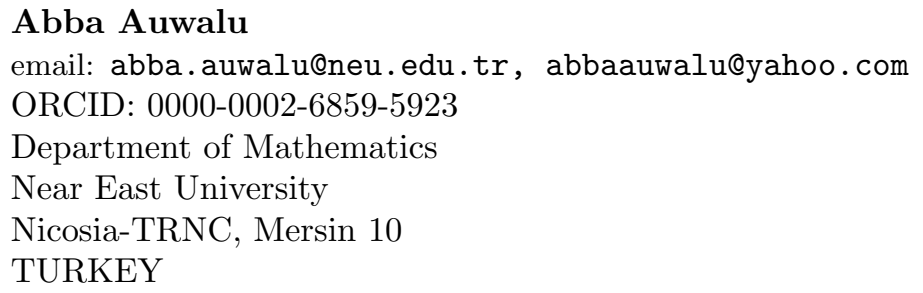

Evren Hinçal

email: evren.hincal@neu.edu.tr, evrenhincal@yahoo.com

Graduate School of Applied Sciences

Near East University

Nicosia-TRNC, Mersin 10

TURKEY

\section{Lakshmi Narayan Mishra*}

email: lakshminarayanmishra04@gmail.com, lakshminarayan.mishra@vit.ac.in ORCID: 0000-0001-7774-7290

School of Advanced Sciences

Vellore Institute of Technology (VIT) University

Vellore 632 014, Tamil Nadu

INDIA

*Corresponding author

Received 21.11.2018

Accepted 08.03.2019 